\title{
Porta-enxertos promissores, alternativos ao limoeiro 'Cravo', nos Tabuleiros Costeiros de Sergipe
}

\author{
Luciana Marques de Carvalho(1), Hélio Wilson Lemos de Carvalho(1), Walter dos Santos Soares Filho(2), \\ Carlos Roberto Martins ${ }^{(3)}$ e Orlando Sampaio Passos ${ }^{(2)}$
}

\begin{abstract}
(1)Embrapa Tabuleiros Costeiros, Avenida Beira Mar, o 3.250, Bairro Jardins, CEP 49025-480 Aracaju, SE, Brasil. E-mail: luciana.carvalho@embrapa.br, helio.carvalho@embrapa.br (2)Embrapa Mandioca e Fruticultura, Rua Embrapa, s/no, Caixa Postal 007, CEP 44380-000 Cruz das Almas, BA, Brasil. E-mail: walter.soares@embrapa.br, orlando.passos@embrapa.br ${ }^{(3)}$ Embrapa Clima Temperado, Rodovia BR-392, Km 78, $9^{\circ}$ Distrito, Monte Bonito, Caixa Postal 403, CEP 96010-971 Pelotas, RS, Brasil. E-mail: carlos.r.martins@embrapa.br
\end{abstract}

Resumo - O objetivo deste trabalho foi identificar porta-enxertos alternativos ao limoeiro 'Cravo' comum, para produção de laranja 'Pêra', nas condições dos Tabuleiros Costeiros de Sergipe. Foram avaliados 43 porta-enxertos quanto a altura, copa, taxa fotossintética, teor de prolina, eficiência produtiva, produção e produtividade por planta. O limoeiro 'Cravo' comum foi considerado como padrão. Os porta-enxertos híbrido TSKC x (LCR x TR) - 059, citrandarim 'Riverside', limoeiro 'Cravo CNPMF - 03' e outros 12 superaram o limoeiro 'Cravo' comum na indução de eficiência produtiva à copa; limoeiro 'Rugoso Vermelho', citrandarim 'Indio' e mais quatro promoveram maior produtividade; TSKC x CTRK - 001 e TSKFL x CTC13 - 012 se destacaram pela magnitude da assimilação de $\mathrm{CO}_{2}$; e TSKC x (LCR x TR) - 040 e TSKC x CTRK - 001 apresentaram maior acúmulo de prolina. São considerados alternativas promissoras os seguintes porta-enxertos: híbrido TSKC $\mathrm{x}$ (LCR x TR) - 059; citrandarins 'Indio', 'Riverside' e 'San Diego'; limoeiros 'Rugoso Vermelho' e 'Cravo CNPMF - 03'; e híbridos TSKC x CTRK - 001, TSKFL x CTC13 - 012, TSKC x (LCR x TR) - 040, TSKC x LHA - 006, TSKC x CTQT1434 - 001, TSKC x CTSW - 058 e TSKFL x CTARG - 028.

Termos para indexação: Citrus, Poncirus, deficit hídrico, híbridos, prolina.

\section{Promising rootstocks alternative to 'Rangpur' lime in the Coastal Tablelands of the state of Sergipe, Brazil}

\begin{abstract}
The objective of this work was to identify alternative rootstocks to 'Rangpur' lime, for production of 'Pêra' orange, under the conditions of the Coastal Tablelands of the state of Sergipe, Brazil. Forty-three rootstocks were evaluated for plant height, canopy, photosynthetic rate, proline content, productive efficiency, yield, and productivity. The 'Rangpur' common lime was considered as standard. The rootstocks TSKC x (LCR x TR) - 059 hybrid, 'Riverside' citrandarin, 'Rangpur CNPMF - 03' lime, and other 12 exceeded 'Rangpur' common lime in inducing crown production efficiency; 'Red Rough' lime, 'Indio' citrandarin, and other four promoted greater productivity; TSKC x CTRK - 001 and TSKFL x CTC13 - 012 stood out for magnitude of $\mathrm{CO}_{2}$ assimilation; and TSKC x (LCR x TR) - 040 and TSKC x CTRK - 001 showed higher proline accumulation. The following rootstocks are considered promising alternatives: TSKC x (LCR x TR) - 059 hybrid; 'Indio', 'Riverside', and 'San Diego' citrandarins; 'Rugoso Vermelho' and 'Rangpur CNPMF - 03' limes; and TSKC x CTRK - 001, TSKFL x CTC13 - 012, TSKC x (LCR x TR) - 040, TSKC x LHA - 006, TSKC x CTQT1434 001, TSKC x CTSW - 058, and TSKFL x CTARG - 028 hybrids.
\end{abstract}

Index terms: Citrus, Poncirus, water deficit, hybrids, proline.

\section{Introdução}

O Brasil é o país com maior área de produção de laranjas doces [Citrus sinensis (L.) Osbeck], com 762.765 mil hectares, mas é o décimo em produtividade (Food and Agriculture Organization of the United Nations, 2012), o que indica a necessidade de adoção de melhores tecnologias. A produção brasileira concentra-se na região Sudeste, seguida pelo Nordeste, responsável por $10 \%$ da produção nacional, com 1,9 milhão de toneladas de frutos, obtidos em 132 mil hectares (Instituto Brasileiro de Geografia e Estatística, 2014). Cerca de $90 \%$ dessa área se encontra nos Tabuleiros Costeiros da Bahia e de Sergipe. A área plantada no Nordeste continua crescendo (Instituto Brasileiro de Geografia e Estatística, 2014), uma vez

Pesq. agropec. bras., Brasília, v.51, n.2, p.132-141, fev. 2016

DOI: 10.1590/S0100-204X2016000200005 
que, até agora, não foi detectada, na região, a ocorrência de "Huanglongbing", importante doença dos citros, relatada pela primeira vez em 2004, em Araraquara, SP (Texeira et al., 2005).

A maioria dos pomares é baseada em plantas enxertadas, em que atributos favoráveis da copa são combinados com aqueles do porta-enxerto. No entanto, um número restrito de variedades-copa é utilizado nos pomares e um menor ainda de porta-enxertos. Entre as variedades-copa mais utilizadas no Nordeste, destaca-se a laranjeira 'Pêra CNPMF D6', em razão do excelente desempenho e da presença de estirpe fraca do Citrus tristeza virus - CTV (Barbosa \& Rodrigues, 2014). O limoeiro (Citrus limonia Osbeck) 'Cravo' é o porta-enxerto mais plantado, por conferir maior vigor, produtividade e longevidade às copas (Cunha Sobrinho et al., 2013). Contudo, esse porta-enxerto é susceptível ao declínio e à morte súbita do citros, além de promover a produção de frutos com qualidade inferior, comparado a porta-enxertos como a tangerineira [C. sunki (Hayata) hort. ex Tanaka] 'Sunki'. Outro fator a considerar é a compatibilidade entre copa e porta-enxerto (Cristofani-Yaly et al., 2007). Sintomas típicos de incompatibilidade incluem o desenvolvimento de uma linha necrótica na região da enxertia, geralmente expressa após 4 a 6 anos (Moraes et al., 2011). Portanto, justifica-se a necessidade de diversificação de porta-enxertos.

Em 2008, em razão da importância da citricultura nos Tabuleiros Costeiros do Nordeste, bem como das limitações e dos desafios à produtividade, a Embrapa iniciou estudos com citros em pomares experimentais. Cabe destacar que, apesar da potencialidade dos Tabuleiros para a citricultura, também há aspectos não favoráveis, como a má distribuição das chuvas, com 70 a $75 \%$ concentradas de abril a setembro, e a ocorrência de uma camada coesa nos solos (Cunha Sobrinho et al., 2013). O horizonte coeso implica na redução da profundidade efetiva do solo e no impedimento à penetração das raízes, o que compromete a produção (Portela et al., 2001). Sintomas de deficiência são comumente observados, uma vez que a demanda evapotranspiratória está entre 900 e $1.200 \mathrm{~mm}$ por ano nas condições brasileiras (Donato et al., 2007) e a maioria dos pomares não é irrigada (Medina et al., 2008). O deficit hídrico induz à redução nas taxas fotossintéticas por meio de limitações estomáticas e não estomáticas. A principal resposta adaptativa das plantas à seca com efeito na produtividade é o ajuste osmótico, proporcionado pelo acúmulo de solutos, como a prolina. Isso permite que as plantas mantenham o turgor foliar necessário à abertura estomática sob baixo potencial hídrico, além de favorecer a absorção de água pelas raízes (Blum, 2009).

Certas características dos porta-enxertos, como padrão de distribuição, crescimento e desenvolvimento das raízes, têm marcante influência na adaptação ao ambiente (Magalhães Filho et al., 2008), o que indica a sua importância na produtividade das plantas.

O objetivo deste trabalho foi identificar portaenxertos alternativos ao limoeiro 'Cravo' comum, para a produção de laranja 'Pêra', nas condições dos Tabuleiros Costeiros de Sergipe.

\section{Material e Métodos}

Para o experimento, utilizou-se copa de laranjeira 'Pêra', clone 'CNPMF D6', enxertada por borbulhia em 43 porta-enxertos (Tabela 1), que compreenderam clones nucelares dos limoeiros (C. limonia) 'Cravo' (LCR) comum, 'Cravo Santa Cruz' (STC), 'CNPMF - 03' e 'CNPMF - 04'; do limoeiro (C. jambhiri Lush) 'Rugoso Vermelho' (LRV); do limoeiro (C. volkameriana V. Ten. \& Pasq.) 'Volkameriano' (LVK); do citrumelo [C. paradisi Macfad. x Poncirus trifoliata (L.) Raf.] 'Swingle' (CTSW); do tangelo (C. paradisi x C. tangerina hort. ex Tanaka) 'Orlando' (OLD); e dos citrandarins (C. sunki x P. trifoliata) 'Indio', 'Riverside' e 'San Diego'. Também foram utilizados clones nucelares de híbridos obtidos pelo Programa de Melhoramento Genético de Citros da Embrapa Mandioca e Fruticultura (PMG Citros): LVK x LCR - 030 e 038; tangerineira 'Sunki' comum (TSKC) x CTSW - 009, 018, 019, 022, 041, 047 e 058; tangerineira 'Sunki' da Flórida (TSKFL) x CTSW - 009; TSKC x citrange $(C$. sinensis $\mathrm{x} P$. trifoliata) 'Troyer' (CTTR) - 008 e 012; TSKC x citrange 'Rusk' (CTRK) - 001; TSKFL x citrange 'Argentina' (CTARG) - 028 e 044; TSKFL $x$ citrange C13 (CTC13) - 012; TSKC x P. trifoliata seleção diploide (TRDP) - 007 e 023; TSKC x [LCR x TR (P. trifoliata)] - 018, 020, 029, 040 e 059; TSKC x citrangequat [Fortunella margarita (Lour.) 'Swingle' x citrange 'Willits'] 'Thomasville' (CTQT1434) - 001; TSKC x CTQT1439 - 026; TSKC x laranjeira 'Hamlin' (LHA) - 006; limoeiro 'Cravo' da Estação Experimental de Limeira (LCREEL) x (LCR x 
Tabela 1. Altura da planta, volume da copa, peso médio do fruto, eficiência produtiva por unidade de volume e produtividade do pomar no quarto ano, em 2013 e acumulada de 2011-2013, de laranjeira (Citrus sinensis) 'Pêra CNPMF D6' sobre 43 porta-enxertos ${ }^{(1)}$.

\begin{tabular}{|c|c|c|c|c|c|c|}
\hline \multirow[t]{2}{*}{ Porta-enxertos ${ }^{(2)}$} & \multirow{2}{*}{$\begin{array}{l}\text { Altura } \\
(\mathrm{m})\end{array}$} & \multirow{2}{*}{$\begin{array}{c}\text { Copa } \\
\left(\mathrm{m}^{3}\right)\end{array}$} & \multirow{2}{*}{$\begin{array}{l}\text { Peso do fruto } \\
\text { (g) }\end{array}$} & \multirow{2}{*}{$\begin{array}{l}\text { Eficiência produtiva } \\
\qquad\left(\mathrm{kg} \mathrm{m}^{-3}\right)\end{array}$} & \multicolumn{2}{|c|}{ Produtividade $\left(\mathrm{kg} \mathrm{ha}^{-1}\right)$} \\
\hline & & & & & 2013 & Acumulada \\
\hline Limoeiro 'Cravo' comum & $197 \mathrm{a}$ & $4,57 \mathrm{a}$ & $210 \mathrm{a}$ & $7,00 \mathrm{a}$ & $13,300 \mathrm{a}$ & 24,931 \\
\hline Limoeiro 'Cravo Santa Cruz' & $177 \mathrm{a}$ & $274 b$ & $200 \mathrm{a}$ & $813 a$ & $10,805 b$ & 22,773 \\
\hline Limoeiro 'Cravo CNPMF - 03' & $190 \mathrm{a}$ & $323 b$ & $250 \mathrm{a}$ & $973 a$ & $10,070 \mathrm{~b}$ & 19,046 \\
\hline Limoeiro 'Cravo CNPMF - 04' & $181 \mathrm{a}$ & $316 b$ & $180 \mathrm{a}$ & $814 \mathrm{a}$ & $10,710 \mathrm{~b}$ & 24,135 \\
\hline Limoeiro 'Volkameriano' & $210 \mathrm{a}$ & $501 \mathrm{a}$ & $180 \mathrm{a}$ & $495 \mathrm{~b}$ & $12,370 \mathrm{~b}$ & 20,950 \\
\hline Limoeiro 'Rugoso Vermelho' & $187 \mathrm{a}$ & $500 \mathrm{a}$ & $210 \mathrm{a}$ & $800 \mathrm{a}$ & $15,535 \mathrm{a}$ & 24,395 \\
\hline Citrandarim 'Indio' & $195 \mathrm{a}$ & $458 \mathrm{a}$ & $200 \mathrm{a}$ & $735 \mathrm{a}$ & $15,370 \mathrm{a}$ & 24,481 \\
\hline Citrandarim 'Riverside' & $195 \mathrm{a}$ & $410 \mathrm{a}$ & $202 a$ & $993 a$ & $11,660 \mathrm{~b}$ & 14,886 \\
\hline Citrandarim 'San Diego' & $162 \mathrm{a}$ & $246 c$ & $200 \mathrm{a}$ & $767 \mathrm{a}$ & $8,560 \mathrm{c}$ & 14,322 \\
\hline Citrumelo 'Swingle' & $170 \mathrm{a}$ & $282 b$ & $190 \mathrm{a}$ & $889 \mathrm{a}$ & $5,835 \mathrm{~d}$ & 7,358 \\
\hline Tangelo 'Orlando' & $178 \mathrm{a}$ & $351 b$ & $210 \mathrm{a}$ & $467 \mathrm{~b}$ & $4,520 \mathrm{~d}$ & 6,160 \\
\hline HTR - 207 & $137 \mathrm{a}$ & $142 \mathrm{c}$ & $193 a$ & $423 b$ & $1,680 \mathrm{~d}$ & 5,451 \\
\hline HTR - 208 & $187 \mathrm{a}$ & $405 \mathrm{a}$ & $200 \mathrm{a}$ & $349 b$ & $6,110 \mathrm{~d}$ & 12,930 \\
\hline TSKC x CTQT1434 - 001 & $200 \mathrm{a}$ & $513 \mathrm{a}$ & $160 \mathrm{a}$ & $800 \mathrm{a}$ & $14,670 \mathrm{a}$ & 17,124 \\
\hline TSKC x CTSW - 041 & $194 \mathrm{a}$ & $523 a$ & $210 \mathrm{a}$ & $600 \mathrm{~b}$ & $14,380 \mathrm{a}$ & 23,104 \\
\hline TSKC $x$ CTSW - 058 & $170 \mathrm{a}$ & $318 b$ & $195 \mathrm{a}$ & $900 \mathrm{a}$ & $13,870 \mathrm{a}$ & 18,677 \\
\hline TSKFL x CTARG - 028 & $186 a$ & $409 a$ & $195 \mathrm{a}$ & $800 \mathrm{a}$ & $13,610 \mathrm{a}$ & 23,530 \\
\hline TSKFL x CTC13 - 012 & $203 a$ & $471 \mathrm{a}$ & $205 \mathrm{a}$ & $545 b$ & $13,105 \mathrm{a}$ & 16,655 \\
\hline LVK x LCR - 038 & $189 \mathrm{a}$ & $443 a$ & $220 \mathrm{a}$ & $515 \mathrm{~b}$ & $12,025 b$ & 24,214 \\
\hline TSKFL x CTTR - 012 & $178 \mathrm{a}$ & $406 a$ & $210 \mathrm{a}$ & $750 \mathrm{a}$ & $11,675 b$ & 16,287 \\
\hline TSKC x CTSW - 047 & $173 a$ & $352 b$ & $215 \mathrm{a}$ & $700 \mathrm{a}$ & $11,210 \mathrm{~b}$ & 12,610 \\
\hline TSKC x CTSW - 019 & $179 \mathrm{a}$ & $388 \mathrm{a}$ & $175 \mathrm{a}$ & $600 \mathrm{~b}$ & $10,165 b$ & 16,720 \\
\hline TSKC $x$ TRDP -023 & $196 a$ & $439 \mathrm{a}$ & $205 \mathrm{a}$ & $650 \mathrm{~b}$ & $9,330 \mathrm{c}$ & 13,554 \\
\hline TSKC $\mathrm{x}$ TRDP -007 & $187 \mathrm{a}$ & $440 \mathrm{a}$ & $200 \mathrm{a}$ & $550 \mathrm{~b}$ & $9,100 \mathrm{c}$ & 13,503 \\
\hline TSKC $x($ LCR $x$ TR $)-059$ & $158 \mathrm{a}$ & $218 \mathrm{c}$ & $195 \mathrm{a}$ & $120 \mathrm{a}$ & $8,555 \mathrm{c}$ & 16,803 \\
\hline TSKC $x($ LCR $x$ TR $)-029$ & $182 \mathrm{a}$ & $328 b$ & $205 \mathrm{a}$ & $700 \mathrm{a}$ & $8,015 \mathrm{c}$ & 13,386 \\
\hline TSKC $x$ (LCR $x$ TR) -040 & $165 \mathrm{a}$ & $285 b$ & $205 \mathrm{a}$ & $700 \mathrm{a}$ & $7,990 \mathrm{c}$ & 15,499 \\
\hline TSKC x CTARG - 044 & $170 \mathrm{a}$ & $279 b$ & $185 \mathrm{a}$ & $700 \mathrm{a}$ & $7,905 \mathrm{c}$ & 10,087 \\
\hline TSKC $x($ LCR $x$ TR $)-020$ & $164 \mathrm{a}$ & $227 \mathrm{c}$ & $195 \mathrm{a}$ & $850 \mathrm{a}$ & $7,855 \mathrm{c}$ & 13,508 \\
\hline MXWL x LHA - 004 & $195 \mathrm{a}$ & $455 \mathrm{a}$ & $154 \mathrm{a}$ & $420 \mathrm{~b}$ & $7,605 \mathrm{c}$ & 16,014 \\
\hline TSKFL x CTTR - 008 & $173 a$ & $303 b$ & $185 \mathrm{a}$ & $450 \mathrm{~b}$ & $7,515 \mathrm{c}$ & 11,890 \\
\hline TSKC x LHA - 006 & $150 \mathrm{a}$ & $220 \mathrm{c}$ & $230 \mathrm{a}$ & $900 \mathrm{a}$ & $7,460 \mathrm{c}$ & 9,367 \\
\hline LVK x LCR - 030 & $178 \mathrm{a}$ & $367 a$ & $170 \mathrm{a}$ & $371 b$ & $7,000 \mathrm{c}$ & 9,919 \\
\hline LRF x (LCR x TR) - 004 & $174 \mathrm{a}$ & $287 \mathrm{~b}$ & $195 \mathrm{a}$ & $407 \mathrm{~b}$ & $6,190 \mathrm{~d}$ & 9,783 \\
\hline MXWL x LHA - 001 & $190 \mathrm{a}$ & $326 \mathrm{~b}$ & $195 \mathrm{a}$ & $396 b$ & $5,895 \mathrm{~d}$ & 9,081 \\
\hline LVK x LCR - 018 & $179 \mathrm{a}$ & $278 b$ & $220 \mathrm{a}$ & $588 \mathrm{~b}$ & $5,750 \mathrm{~d}$ & 7,900 \\
\hline TSKC x CTSW - 022 & $174 \mathrm{a}$ & $324 b$ & $170 \mathrm{a}$ & $500 \mathrm{~b}$ & $5,490 \mathrm{~d}$ & 5,490 \\
\hline TSKC x CTRK - 001 & $182 \mathrm{a}$ & $313 b$ & $195 \mathrm{a}$ & $442 b$ & $5,190 \mathrm{~d}$ & 7,084 \\
\hline LCREEL x (LCR x TR) - 001 & $153 \mathrm{a}$ & $201 \mathrm{c}$ & $193 \mathrm{a}$ & $551 \mathrm{~b}$ & $5,100 \mathrm{~d}$ & 5,100 \\
\hline TSKFL x CTSW - 009 & $166 \mathrm{a}$ & $344 \mathrm{~b}$ & $195 \mathrm{a}$ & $400 \mathrm{~b}$ & $4,930 \mathrm{~d}$ & 4,930 \\
\hline TSKC $x($ LCR $x$ TR $)-018$ & $170 \mathrm{a}$ & $273 b$ & $225 \mathrm{a}$ & $550 \mathrm{~b}$ & $4,885 \mathrm{~d}$ & 4,885 \\
\hline TSKC x CTSW - 018 & $150 \mathrm{a}$ & $159 \mathrm{c}$ & $195 \mathrm{a}$ & $650 \mathrm{~b}$ & $4,600 \mathrm{~d}$ & 4,600 \\
\hline TSKC x CTQT1439 - 026 & $156 \mathrm{a}$ & $278 \mathrm{~b}$ & $235 \mathrm{a}$ & $450 \mathrm{~b}$ & $4,475 \mathrm{~d}$ & 11,389 \\
\hline Média & 177 & 344 & 199 & 641 & 8,855 & 13,799 \\
\hline CV (\%) & 8,9 & 15,1 & 8,2 & 9,2 & 13,2 & - \\
\hline
\end{tabular}

${ }^{(1)}$ Médias seguidas de letras iguais, nas colunas, não diferem pelo teste de Scott-Knott, a 5\% de probabilidade. Os dados representam médias de avaliações em três blocos de duas plantas por tratamento, o que totalizou seis repetições em plantios estabelecidos no campo, em agosto de 2009. (2)Limoeiros 'Cravo' a 'Cravo CNPMF - 04', C. limonia; limoeiro 'Volkameriano', C. volkameriana; limoeiro 'Rugoso Vermelho', C. jambhiri; citrandarins 'Indio' a 'San Diego', C. sunki $\mathrm{x}$ Poncirus trifoliata; citrumelo 'Swingle', C. paradisi x P. trifoliata; tangelo 'Orlando', C. paradisi x C. tangerina; HTR, híbrido trifoliado; TSKC, tangerineira (C. sunki) 'Sunki' comum; CTQT, citrangequat [Fortunella margarita 'Swingle' x citrange (C. sinensis x P. trifoliata) 'Willits'] 'Thomasville'; CTSW, citrumelo 'Swingle'; TSKFL, tangerineira 'Sunki', seleção da Flórida; CTARG, citrange 'Argentina'; CTC13, citrange C13; LVK, limoeiro 'Volkameriano'; LCR, limoeiro 'Cravo'; CTTR, citrange 'Troyer'; TRDP, P. trifoliata, seleção diploide; TR, P. trifoliata; LRF, limoeiro 'Rugoso Vermelho'; MXWL, mexerica (C. deliciosa), seleção 'Willow Leaf'; LHA, laranjeira (C. sinensis) 'Hamlin'; CTRK, citrange 'Rusk'; e LCREEL, limoeiro 'Cravo', seleção da Estação Experimental de Limeira. 
TR) - 001; limoeiro 'Rugoso' da Flórida (LRF) x (LCR $\mathrm{x}$ TR) - 004; mexerica (C. deliciosa Ten.) 'Willow Leaf' (MXWL) x LHA - 001 e 004; e híbrido trifoliado (HTR) - 207 e 208. Os tratamentos consistiram das combinações copa/porta-enxerto e foram avaliados no delineamento de blocos ao acaso, com três repetições e três plantas por parcela. Uma linha de laranja 'Pêra' enxertada em limão 'Cravo' comum foi utilizada como bordadura geral na periferia dos tratamentos.

As mudas obtidas, em viveiro protegido com tela antiafídeos, seguindo os padrões comerciais de produção, foram plantadas no espaçamento de 5,0x3,0 m, em agosto de 2009, em pomar situado no


a $109 \mathrm{~m}$ de altitude). A área experimental apresenta solo Argissolo Acinzentado eutrófico Tb, A fraco, com textura média/argilosa, e relevo predominantemente plano (Portela et al., 2001). O clima é quente e úmido, tipo As, de acordo com a classificação de Köppen, com precipitação média anual de $1.263,6 \mathrm{~mm}$, concentrada nos meses de abril a setembro. A precipitação mensal nos anos da condução do experimento e da série histórica são apresentados na Figura 1. A adubação e os tratos culturais foram os recomendados para citros (Mattos Junior et al., 2005), de acordo com análise de solo.

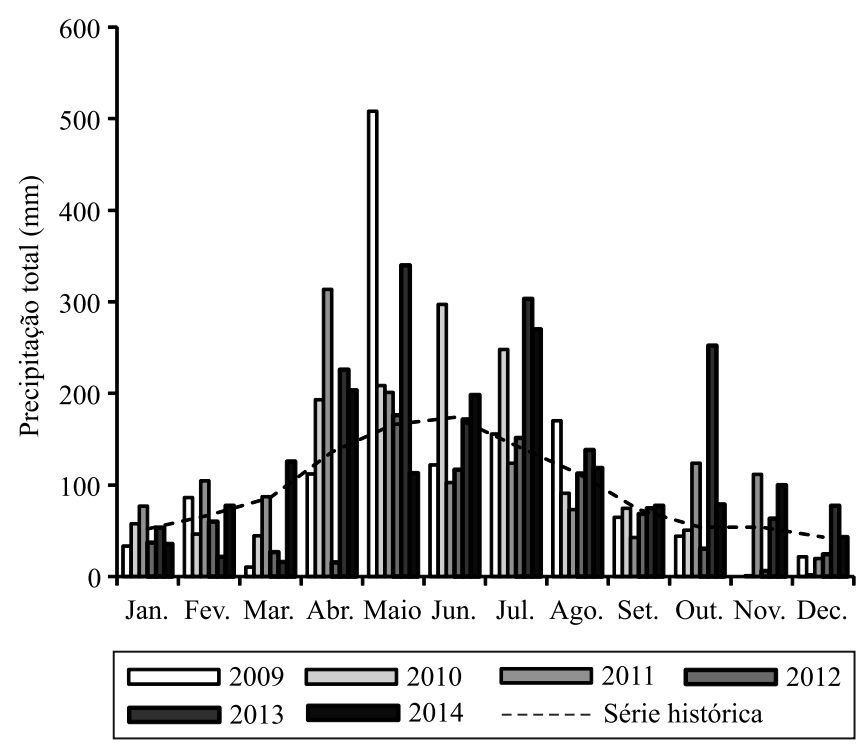

Figura 1. Precipitação mensal nos anos agrícolas de 20092014 e série histórica, no campo experimental da Embrapa, situado em Umbaúba, SE.
As taxas de assimilação de dióxido de carbono (A), de evapotranspiração (E) e de condutância estomática ao vapor de água (gs) foram determinadas em três datas, distanciadas de 1 semana, o que totalizou seis repetições, de março a abril de 2012 , entre 9 h00 e 11 h00. As avaliações foram feitas em uma folha madura, exposta ao sol, em posição intermediária do caule, em duas plantas de cada tratamento, por meio de analisador de gases portátil a infravermelho, modelo LCPro+, ADC (BioScientific Ltda., Hoddesdon, Hertfordshire, Reino Unido), com irradiância de $1.000 \mu \mathrm{mol} \mathrm{m} \mathrm{m}^{-2} \mathrm{~s}^{-1}$ de fótons. Com esses dados, estimaram-se a eficiência instantânea no uso da água, a partir da razão $\mathrm{A} / \mathrm{E}$, e a eficiência intrínseca de uso da água para assimilação de $\mathrm{CO}_{2}$, a partir da estimativa da razão A/gs (Medina \& Machado, 1998). No quarto ano do pomar, em 2013, foram determinados, de acordo com Auler et al. (2008a): altura total da planta $(\mathrm{H})$; volume da copa, estimado com base no raio da copa (R) e na altura, a partir da equação $V=2 / 3\left(\pi \mathrm{R}^{2} \mathrm{H}\right)$; peso médio de frutos por planta; eficiência produtiva por unidade de volume; e produtividade, a partir da massa fresca e da área de produção. A produtividade acumulada de frutos foi estimada com base na produção obtida em 2011, 2012 e 2013. Em agosto de 2014, o teor de prolina foi determinado como descrito em Bates et al. (1973), em amostras de $25 \mathrm{mg}$ da folha expandida, na região mais apical, exposta ao sol, retiradas de 17 dos 43 tratamentos, selecionados com base no seu desempenho. Todos os dados foram submetidos à análise de variância e ao teste de comparação de médias de Scott-Knott, a 5\% de probabilidade, por meio do programa computacional Sisvar, versão 5.3 (Universidade Federal de Lavras, Lavras, MG). Os dados obtidos a partir de cálculos numéricos foram transformados em raiz quadrada.

\section{Resultados e Discussão}

Dos 43 porta-enxertos avaliados no quarto ano, 15 induziram eficiência produtiva numericamente superior à do limoeiro 'Cravo' comum. Entre esses, TSKC x (LCR x TR) - 059, citrandarim 'Riverside', limoeiro 'Cravo CNPMF - 03', TSKC x CTSW - 058 e TSKC x LHA - 006 destacaram-se por induzir eficiência produtiva igual ou superior a $9,0 \mathrm{~kg} \mathrm{~m}^{-3}$ (Tabela 1). Entretanto, as médias obtidas não diferiram significativamente daquelas para plantas sobre os limoeiros 'Cravo' comum, 'Cravo Santa Cruz', 'Cravo 
CNPMF - 04' e 'Rugoso Vermelho'; o citrumelo 'Swingle'; os citrandarins 'Indio' e 'San Diego'; e os híbridos TSKC x CTQT1434 - 001, TSKFL x CTARG - 028, TSKC x CTTR - 012, TSKC x CTSW - 047, TSKC $\times($ LCR $\times$ TR) -029 , TSKC $\times($ LCR $\times$ TR) 040, TSKC x CTARG - 044 e TSKC x (LCR x TR) - 020. Ressalta-se que, até o momento, não foram verificados sintomas visuais de incompatibilidade em nenhuma das combinações avaliadas, apesar de haver relatos de incompatibilidade entre a copa de laranjeira 'Pêra' e alguns porta-enxertos, especialmente híbridos de $P$. trifoliata (Moraes et al., 2011). Porém, a incompatibilidade nem sempre se expressa prontamente, o que implica na necessidade de um número maior de anos de observações para se chegar a conclusões definitivas. Cristofani-Yaly et al. (2007) constataram o desenvolvimento de sintomas típicos de incompatibilidade, aos 4 anos de idade, em apenas três dos 111 híbridos de 'Sunki' vs. P. trifoliata enxertados em 'Pêra', e Schinor et al. (2013), em dois dos 42 híbridos de 'Sunki' vs. P. trifoliata avaliados aos 7 anos de idade. Isso indica que sintomas de incompatibilidade nessas plantas podem ainda ser expressos.

A superioridade do híbrido TSKC x (LCR x TR) - 059 na indução de maior eficiência produtiva e produtividade também foi observada por Ramos et al. (2015), ao avaliar a copa de laranjeira 'Valência', em plantas com 2, 3 e 4 anos de idade, em um conjunto de mais de 40 porta-enxertos, cultivados no município de Colômbia, SP. Esse resultado é indicativo da precocidade produtiva desse porta-enxerto. Além disso, a maior eficiência produtiva não coincidiu, em todos os casos, com maior produtividade de frutos nos primeiros anos, como verificado para os porta-enxertos TSKC x (LCR x TR) - 059, TSKC x LHA - 006, TSKC x (LCR x TR) - 020 e citrandarim 'San Diego' (Tabela 2).

A precocidade produtiva, indicada pela maior produtividade no quarto ano, foi determinada nas plantas enxertadas sobre: os limoeiros 'Cravo' comum e 'Rugoso Vermelho'; o citrandarim 'Indio'; e os híbridos TSKFL x CTARG - 028, TSKC x CTQT1434 - 001 e TSKC x CTSW - 058 (Tabela 1). Observou-se precocidade nas plantas com maiores copas, exceto naquelas enxertadas em TSKC $\mathrm{x}$ CTSW - 058 (Tabela 1), à semelhança do que ocorre em laranjeira 'Valência' (Blumer, 2005). Plantas com maior biomassa foliar têm maior potencial de absorver água e colocar a raiz em contato com nutrientes (Rodriguez-Gamir et al., 2010). No entanto, entre os 20 porta-enxertos com maior eficiência produtiva, apenas em sete constataram-se maiores copas: nos limoeiros 'Cravo' e 'Rugoso Vermelho'; nos citrandarins 'Indio' e 'Riverside'; e nos híbridos TSKFL x CTARG - 028, TSKC x CTQT1434 - 001 e TSKC x CTTR - 012 (Tabela 1). Auler et al. (2008) sugeriram que porta-enxertos que induzem copas menores e eficiência produtiva superior, como TSKC x (LCR x TR) - 059, TSKC x LHA - 006, TSKC x (LCR x TR) - 020 e o citrandarim 'San Diego', são interessantes, pois possibilitam o incremento na produção com o adensamento do plantio. Além disso, a menor altura da planta favorece a eficiência na inspeção de controle de doenças e pragas, bem como a redução no custo das colheitas (Blumer, 2005). Verificou-se menor porte com os quatro porta-enxertos supracitados, embora as plantas não tenham diferido significativamente quanto à altura. Entre as seleções de 'Cravo' avaliadas, maior eficiência produtiva associada à copa de menor porte foi obtida com o clone 'CNPMF - 03'.

Outros porta-enxertos, como 'Cravo CNPMF - 04' e LVK x LCR - 038, destacaram-se por favorecer maior produtividade acumulada de 2011-2013, apesar da baixa produtividade no quarto ano. $\mathrm{O}$ peso médio de fruto, entretanto, não variou significativamente entre as combinações avaliadas (Tabela 1). Esses dados indicaram que a variação na produtividade se deveu mais ao número de frutos por planta; também confirmaram a irregularidade e a alternância de safras para a copa de 'Pêra CNPMF D6', de acordo com o relatado por Blumer (2005) e Auler et al. (2008), ao avaliar copa de laranjeira 'Valência' nas condições de São Paulo.

A taxa de assimilação de $\mathrm{CO}_{2}$ variou de 1,34 $\mu \mathrm{mol} \mathrm{m}{ }^{-2} \mathrm{~s}^{-1}$ de $\mathrm{CO}_{2}$, nas plantas sobre o porta-enxerto 'Volkameriano' que não diferiram daquelas sobre 'Cravo' comum, a 25,24 $\mu \mathrm{mol} \mathrm{m}{ }^{-2} \mathrm{~s}^{-1} \mathrm{de} \mathrm{CO}_{2}$, nas plantas sobre TSKFL x CTC13 - 012 (Tabela 2). Conforme Flexas et al. (2006), o principal fator responsável pela menor assimilação de $\mathrm{CO}_{2}$ nas plantas de citros sob deficiência hídrica é a reduzida disponibilidade de $\mathrm{CO}_{2}$ atmosférico, decorrente de limitação na difusão por estômatos e mesófilo. Contudo, constatou-se que as altas taxas de assimilação de $\mathrm{CO}_{2}$ observadas nas plantas sobre TSKFL x CTC13 - 012 e TSKC 
Tabela 2. Rendimento no quarto ano $\left(\mathrm{kg} \mathrm{ha}^{-1}\right)$ e taxas transpiratórias $(\mathrm{E})$, de assimilação líquida de $\mathrm{CO}_{2}(\mathrm{~A})$, de eficiência instantânea no uso da água (A/E), de condutância estomática (gs) em folhas individuais e de eficiência intrínseca no uso da água (A/gs) de laranjeira (Citrus sinensis) 'Pêra CNPMF D6' sobre 43 porta-enxertos ${ }^{(1)}$.

\begin{tabular}{|c|c|c|c|c|c|}
\hline Porta-enxertos $^{(2)}$ & $\begin{array}{c}\mathrm{E} \\
\left(\mu \mathrm{mol} \mathrm{m}{ }^{-2} \mathrm{~s}^{-1} \text { de } \mathrm{H}_{2} \mathrm{O}\right) \\
\end{array}$ & $\begin{array}{c}\mathrm{A} \\
\left(\mu \mathrm{mol} \mathrm{m}-2 \mathrm{~s}^{-1}{\left.\mathrm{de} \mathrm{CO}_{2}\right)}\right.\end{array}$ & $\mathrm{A} / \mathrm{E}$ & $\begin{array}{c}\mathrm{gs} \\
\left(\mathrm{mol} \mathrm{m}^{-2} \mathrm{~s}^{-1} \mathrm{de}_{2} \mathrm{O}\right)\end{array}$ & $\mathrm{A} / \mathrm{gs}$ \\
\hline Limoeiro 'Cravo' comum & $1,053 \mathrm{c}$ & $2,19 \mathrm{e}$ & $2,090 \mathrm{c}$ & $0,050 \mathrm{c}$ & $43,80 \mathrm{c}$ \\
\hline Limoeiro 'Cravo Santa Cruz' & $1,163 \mathrm{c}$ & $2,46 \mathrm{e}$ & $2,108 \mathrm{c}$ & $0,055 \mathrm{c}$ & $46,06 \mathrm{c}$ \\
\hline Limoeiro 'Cravo CNPMF - 03' & $1,513 \mathrm{a}$ & $2,11 \mathrm{e}$ & $1,558 \mathrm{c}$ & $0,065 b$ & $35,97 \mathrm{c}$ \\
\hline Limoeiro 'Cravo CNPMF - 04' & $1,633 \mathrm{a}$ & $3,80 \mathrm{~d}$ & $2,338 \mathrm{c}$ & $0,078 b$ & $48,33 \mathrm{c}$ \\
\hline Limoeiro 'Volkameriano' & $0,880 \mathrm{c}$ & $1,34 \mathrm{e}$ & $1,665 \mathrm{c}$ & $0,040 \mathrm{c}$ & $39,40 \mathrm{c}$ \\
\hline Limoeiro 'Rugoso Vermelho' & $1,150 \mathrm{c}$ & $4,85 \mathrm{c}$ & $4,295 b$ & $0,055 \mathrm{c}$ & $90,84 \mathrm{~b}$ \\
\hline Citrandarim 'Indio' & $0,958 \mathrm{c}$ & $2,35 \mathrm{e}$ & $2,433 \mathrm{c}$ & $0,043 \mathrm{c}$ & $57,36 \mathrm{c}$ \\
\hline Citrandarim 'Riverside' & $1,255 b$ & $3,62 d$ & $2,678 \mathrm{c}$ & $0,055 \mathrm{c}$ & $61,28 \mathrm{c}$ \\
\hline Citrandarim 'San Diego' & $1,138 \mathrm{c}$ & $2,58 \mathrm{e}$ & $2,223 \mathrm{c}$ & $0,050 \mathrm{c}$ & $51,30 \mathrm{c}$ \\
\hline Citrumelo 'Swingle' & $0,873 \mathrm{c}$ & $1,97 \mathrm{e}$ & $2,308 \mathrm{c}$ & $0,040 \mathrm{c}$ & $51,92 \mathrm{c}$ \\
\hline Tangelo 'Orlando' & $1,453 b$ & $4,09 \mathrm{c}$ & $2,753 \mathrm{c}$ & $0,073 b$ & $53,95 \mathrm{c}$ \\
\hline HTR - 207 & $1,085 \mathrm{c}$ & $2,24 \mathrm{e}$ & $1,985 \mathrm{c}$ & $0,045 \mathrm{c}$ & $47,75 \mathrm{c}$ \\
\hline HTR - 208 & $1,258 \mathrm{~b}$ & $2,84 \mathrm{e}$ & $2,140 \mathrm{c}$ & $0,058 \mathrm{c}$ & $48,43 \mathrm{c}$ \\
\hline TSKC x CTQT1434 - 001 & $0,993 \mathrm{c}$ & $2,48 \mathrm{e}$ & $2,493 \mathrm{c}$ & $0,048 \mathrm{c}$ & $51,86 \mathrm{c}$ \\
\hline TSKC X CTSW - 041 & $1,205 \mathrm{c}$ & $3,19 \mathrm{~d}$ & $2,835 \mathrm{c}$ & $0,060 \mathrm{c}$ & $56,73 \mathrm{c}$ \\
\hline TSKC x CTSW - 058 & $0,965 \mathrm{c}$ & $3,70 \mathrm{~d}$ & $3,988 \mathrm{~b}$ & $0,050 \mathrm{c}$ & $77,78 b$ \\
\hline TSKFL x CTARG - 028 & $1,115 \mathrm{c}$ & $3,44 d$ & $3,143 b$ & $0,053 \mathrm{c}$ & $66,23 \mathrm{~b}$ \\
\hline TSKFL x CTC13 - 012 & $1,283 b$ & $25,24 \mathrm{a}$ & $25,880 \mathrm{a}$ & $0,063 \mathrm{c}$ & $506,73 \mathrm{a}$ \\
\hline LVK x LCR - 038 & $1,380 \mathrm{~b}$ & $3,95 \mathrm{c}$ & $2,838 \mathrm{c}$ & $0,060 \mathrm{c}$ & $67,16 \mathrm{~b}$ \\
\hline TSKC x CTTR - 012 & $1,290 \mathrm{~b}$ & $3,29 \mathrm{~d}$ & $2,725 \mathrm{c}$ & $0,063 \mathrm{c}$ & $62,87 \mathrm{c}$ \\
\hline TSKC x CTSW - 047 & $1,730 \mathrm{a}$ & $5,30 \mathrm{c}$ & $3,023 \mathrm{c}$ & $0,088 b$ & $59,63 \mathrm{c}$ \\
\hline TSKC x CTSW - 019 & $1,610 \mathrm{a}$ & $6,93 \mathrm{~b}$ & $4,300 \mathrm{~b}$ & $0,083 b$ & $84,28 b$ \\
\hline TSKC x TRDP - 023 & $1,565 \mathrm{a}$ & $8,83 \mathrm{~b}$ & $5,658 \mathrm{~b}$ & $0,088 \mathrm{~b}$ & $101,80 \mathrm{~b}$ \\
\hline TSKC $x$ TRDP - 007 & $1,095 \mathrm{c}$ & $4,31 \mathrm{c}$ & $3,998 \mathrm{~b}$ & $0,055 \mathrm{c}$ & $79,44 \mathrm{~b}$ \\
\hline TSKC x (LCR x TR) - 059 & $1,198 \mathrm{c}$ & $4,87 \mathrm{c}$ & $3,930 \mathrm{~b}$ & $0,060 \mathrm{c}$ & $80,41 \mathrm{~b}$ \\
\hline TSKC x (LCR x TR) - 029 & $1,513 \mathrm{a}$ & $5,57 \mathrm{c}$ & $3,645 b$ & $0,078 b$ & $70,78 \mathrm{~b}$ \\
\hline TSKC $x($ LCR $x$ TR $)-040$ & $1,940 \mathrm{a}$ & $8,52 b$ & $4,380 \mathrm{~b}$ & $0,120 \mathrm{a}$ & $71,04 \mathrm{~b}$ \\
\hline TSKC x CTARG - 044 & $1,028 \mathrm{c}$ & $3,11 \mathrm{~d}$ & $3,073 \mathrm{c}$ & $0,053 \mathrm{c}$ & $60,17 \mathrm{c}$ \\
\hline TSKC $x($ LCR $x$ TR $)-020$ & $1,345 b$ & $3,76 \mathrm{~d}$ & $2,643 \mathrm{c}$ & $0,068 \mathrm{~b}$ & $52,86 \mathrm{c}$ \\
\hline MXWL x LHA - 004 & $0,993 \mathrm{c}$ & $2,94 d$ & $2,960 \mathrm{c}$ & $0,048 \mathrm{c}$ & $63,43 b$ \\
\hline TSKC x CTTR - 008 & $1,083 \mathrm{c}$ & $4,62 \mathrm{c}$ & $4,428 b$ & $0,060 \mathrm{c}$ & $77,01 \mathrm{~b}$ \\
\hline TSKC x LHA - 006 & $0,988 \mathrm{c}$ & $3,00 \mathrm{~d}$ & $2,985 \mathrm{c}$ & $0,045 \mathrm{c}$ & $65,12 b$ \\
\hline LVK x LCR - 030 & $1,085 \mathrm{c}$ & $2,04 \mathrm{e}$ & $1,843 \mathrm{c}$ & $0,050 \mathrm{c}$ & $41,17 \mathrm{c}$ \\
\hline LRF x (LCR x TR) - 004 & $1,285 b$ & $3,85 \mathrm{~d}$ & $2,685 \mathrm{c}$ & $0,060 \mathrm{c}$ & $57,97 \mathrm{c}$ \\
\hline MXWL x LHA - 001 & $0,793 \mathrm{c}$ & $1,58 \mathrm{e}$ & $1963 c$ & $0,035 \mathrm{c}$ & $45,00 \mathrm{c}$ \\
\hline LVK x LCR - 018 & $1,048 \mathrm{c}$ & $2,50 \mathrm{e}$ & $2,388 \mathrm{c}$ & $0,048 \mathrm{c}$ & $52,93 \mathrm{c}$ \\
\hline TSKC x CTSW - 022 & $1,118 \mathrm{c}$ & $4,97 \mathrm{c}$ & $4,395 b$ & $0,053 \mathrm{c}$ & $92,98 b$ \\
\hline TSKC x CTRK - 001 & $1,070 \mathrm{c}$ & $23,37 \mathrm{a}$ & $24,850 \mathrm{a}$ & $0,050 \mathrm{c}$ & $504,21 \mathrm{a}$ \\
\hline LCREEL x (TR x LCR) - 001 & $1,008 \mathrm{c}$ & $3,36 \mathrm{~d}$ & $3,585 \mathrm{~b}$ & $0,048 \mathrm{c}$ & $74,12 b$ \\
\hline TSKFL x CTSW - 009 & $0,810 \mathrm{c}$ & $2,41 \mathrm{e}$ & $2,973 \mathrm{c}$ & $0,040 \mathrm{c}$ & $59,77 \mathrm{c}$ \\
\hline TSKC x (LCR x TR) - 018 & $1,403 b$ & $5,23 \mathrm{c}$ & $3,773 b$ & $0,073 b$ & $73,26 \mathrm{~b}$ \\
\hline TSKC x CTS - 018 & $1,270 \mathrm{~b}$ & $4,66 \mathrm{c}$ & $3,643 b$ & $0,063 \mathrm{c}$ & $73,69 b$ \\
\hline TSKC x CTQT 1439 - 026 & $1,393 \mathrm{~b}$ & $3,97 \mathrm{~d}$ & $2,775 \mathrm{c}$ & $0,075 b$ & $51,52 \mathrm{c}$ \\
\hline Média geral & 1,205 & 4,48 & 3,850 & 0,058 & 78,85 \\
\hline CV (\%) & 10,230 & 12,96 & 19,33 & 12,04 & 17,32 \\
\hline
\end{tabular}

${ }^{(1)}$ Médias seguidas de letras iguais, nas colunas, não diferem pelo teste de Scott-Knott, a 5\% de probabilidade. Os dados representam médias de três avaliações realizadas em março/abril de 2012, em duas plantas por tratamento, o que totalizou seis repetições nas mesmas condições de temperatura e radiação incidente em plantios estabelecidos em agosto de 2009. (2)Limoeiros 'Cravo' a 'Cravo CNPMF - 04', C. limonia; limoeiro 'Volkameriano', C. volkameriana; limoeiro 'Rugoso Vermelho', C. jambhiri; citrandarins 'Indio' a 'San Diego', C. sunki x Poncirus trifoliata; citrumelo 'Swingle', C. paradisi x P. trifoliata; tangelo 'Orlando', C. paradisi x C. tangerina; HTR, híbrido trifoliado; TSKC, tangerineira (C. sunki) 'Sunki' comum; CTQT, citrangequat [Fortunella margarita 'Swingle' x citrange (C. sinensis x P. trifoliata) 'Willits'] 'Thomasville'; CTSW, citrumelo 'Swingle'; TSKFL, tangerineira 'Sunki', seleção da Flórida; CTARG, citrange 'Argentina'; CTC13, citrange C13; LVK, limoeiro 'Volkameriano'; LCR, limoeiro 'Cravo'; CTTR, citrange 'Troyer'; TRDP, P. trifoliata, seleção diploide; TR, P. trifoliata; LRF, limoeiro 'Rugoso Vermelho'; MXWL, mexerica (C. deliciosa), seleção 'Willow Leaf'; LHA, laranjeira (C. sinensis) 'Hamlin'; CTRK, citrange 'Rusk'; e LCREEL, limoeiro 'Cravo', seleção da Estação Experimental de Limeira. 
$\mathrm{x}$ CTRK - 001 não coincidiram com as altas taxas de condutância e de evapotranspiração, mas com a alta eficiência no uso da água para assimilação de $\mathrm{CO}_{2}$ (Tabela 2), o que sugere maior tolerância à seca. As plantas enxertadas em TSKC x (LCR x TR) - 040, TSKC x TRDP - 023 e TSKC x CTSW 019 apresentaram altas taxas de assimilação de $\mathrm{CO}_{2}$, embora inferiores àquelas observadas para as plantas citadas anteriormente. A superioridade e a magnitude da condutância estomática das plantas sobre TSKC $\mathrm{x}$ (LCR x TR) - 040 revelam maior abertura estomática e estão em consonância com o maior acúmulo de prolina (Figura 2). No entanto, o teor desta não diferiu daquele obtido nas plantas sobre TSKC x CTRK - 001,
TSKC x TRDP - 023, limoeiro 'Cravo' comum, tangelo 'Orlando' e TSKC x (LCR x TR) - 020.

A condutância estomática da maioria das plantas ficou na faixa de 0,05 a $0,10 \mu \mathrm{mol} \mathrm{m}^{-2} \mathrm{~s}^{-1} \mathrm{de}_{2} \mathrm{O}$, na qual a fotossíntese é limitada principalmente pela restrição na difusão de $\mathrm{CO}_{2}$ (Flexas et al., 2006). Nas plantas com menores condutâncias, como as enxertadas em MXWL x LHA, pressupõe-se a ocorrência de danos metabólicos (Campos et al., 2011). As médias de condutância determinadas foram semelhantes às relatadas por Suassuna et al. (2012) para laranjeiras 'Valência' em deficit hídrico. Magalhães Filho et al. (2008) verificaram, em laranjeiras 'Valência' sobre 'Cravo' e trifoliata, condutância estomática próxima à



Figura 2. Teor de prolina em folhas de laranjeira (Citrus sinensis) 'Pêra CNPMF D6' removidas a partir do terço superior das plantas de 17 porta-enxertos, plantados em agosto de 2009 e amostrados em agosto de 2014, em Umbaúba, SE. Médias seguidas de letras iguais sobre as colunas não diferem pelo teste de Scott-Knott, a 5\% de probabilidade. TSKC, tangerineira (C. sunki) 'Sunki' comum; LHA, laranjeira (C. sinensis) 'Hamlin'; CTQT, citrangequat [Fortunella margarita 'Swingle' $\mathrm{x}$ citrange (C. sinensis x Poncirus trifoliata) 'Willits'] 'Thomasville'; TR, P. trifoliata; LCR, limoeiro 'Cravo'; limoeiro 'Rugoso Vermelho', C. jambhiri; tangelo 'Orlando', C. paradisi x C. tangerina; CTRK, citrange 'Rusk'; 'Cravo', C. limonia; TRDP, $P$. trifoliata, seleção diploide; citrandarins 'Riverside' e 'Indio', C. sunki x P. trifoliata; TSKFL, tangerineira 'Sunki', seleção da Flórida; e CTC13, citrange C13. 
zero, ou seja, fechamento estomático após 12 dias de interrupção na irrigação; segundo os autores, este foi o primeiro mecanismo de defesa acionado. Christmann et al. (2007) concluíram que, em arbóreas, como o citros, a rapidez na resposta estomática deve-se à ação de forças hidráulicas geradas no sistema capilar das plantas em resposta ao deficit hídrico no solo. Já Campos et al. (2011), ao avaliar plantas de citrumelo 'Swingle' transformadas, observaram condutância baixa e altas taxas de assimilação de $\mathrm{CO}_{2}$, e sugeriram a participação de um mecanismo de proteção adicional, em que a prolina age como agente estabilizador de proteínas.

Após período seco prolongado, como o observado no verão de 2012-2013, o limoeiro 'Cravo' comum induziu à baixa perda de água por evapotranspiração e à grande acúmulo de prolina na copa de 'Pêra' (Figura 2), o que está de acordo com a alta tolerância à seca atribuída ao limoeiro 'Cravo' sobre copa de laranjeira 'Valência' (Medina \& Machado, 1998). Porém, no presente trabalho, as taxas de assimilação de $\mathrm{CO}_{2}$ e de eficiência de uso da água na assimilação foram baixas e inferiores àquelas das plantas sobre TSKC x CTRK - 001, TSKFL x CTC13 - 012 e TSKC $x$ (TR x LCR) - 040, o que indica que essas plantas ainda são mais tolerantes do que o 'Cravo' comum ao deficit prolongado. Conforme Magalhães Filho et al. (2008), a tolerância do limoeiro 'Cravo' deve-se, principalmente, a características inerentes ao sistema radicular, em função de seu crescimento ativo mesmo sob deficit hídrico. Além disso, Medina \& Machado (1998) relataram que as plantas de 'Cravo' demoram a se recuperar após deficit severo, possivelmente pelo comprometimento da condutividade hidráulica.

O híbrido TSKC x (LCR x TR) - 059, por sua vez, proporcionou, à copa de 'Pêra', reduzida perda de água por evapotranspiração e eficiência no uso da água para assimilação de $\mathrm{CO}_{2}$ acima da média geral. Esse desempenho provavelmente deveuse tanto à pequena área evapotranspiratória e à reduzida abertura estomática quanto ao provável ajuste osmótico decorrente do acúmulo de prolina. Os porta-enxertos TSKC x CTRK - 001 e TSKFL $x$ CTC13 - 012, seguidos por TSKC x (TR x LCR) 040, além de baixa evapotranspiração, propiciaram altas médias de assimilação de $\mathrm{CO}_{2}$ e de eficiência de uso da água. Com base nesse fato, sugere-se que induziram maior tolerância à seca que o limoeiro 'Cravo' comum.

Os teores de prolina determinados foram superiores aos relatados por Molinari et al. (2004) e Campos et al. (2011), em outras copas, na ausência de estresses ambientais. Segundo Nolte et al. (1997), teores de até $100 \mu \mathrm{mol} \mathrm{g}{ }^{-1}$ de prolina são naturalmente presentes em citros, em razão de processo constitutivo. Também têm sido verificados incrementos, em resposta à deficiência hídrica, associados ao ajustamento osmótico, como forma de reduzir a perda de água por transpiração e manter o crescimento e a produtividade das plantas (Szabados \& Savoure, 2010; Campos et al., 2011; Carvalho et al., 2013). Ao se considerar os níveis extremamente altos constatados em citros, funções adicionais, como proteção oxidativa, têm sido recomendadas (Szabados \& Savoure, 2010).

Os limoeiros 'Cravo' comum, 'Cravo Santa Cruz' e 'Rugoso Vermelho'; os citrandarins 'Indio' e 'San Diego'; e os híbridos TSKC x CTSW - 058, TSKFL x CTARG - 028, TSKC x (LCR x TR) - 059, TSKC x CTARG - 044 e TSKC x LHA - 006, além de induzirem eficiência produtiva igual ou superior a $7,0 \mathrm{~kg} \mathrm{~m}^{-3} \mathrm{de}$ frutos de copa, destacaram-se por propiciar menor perda de água por evapotranspiração, em condição de deficit hídrico.

\section{Conclusões}

1. Os porta-enxertos híbridos TSKC x (LCR x TR) - 059, TSKC x LHA - 006, TSKC x (LCR x TR) - 020 e citrandarim (Citrus sunki vs. Poncirus trifoliata) 'San Diego' são alternativas ao limoeiro (C. limonia) 'Cravo' comum, por induzirem à formação de copa de laranjeira (C. sinensis) 'Pêra' menor e com elevada eficiência produtiva nos primeiros anos.

2. Os porta-enxertos híbridos TSKC x CTRK 001, TSKFL x CTC13 - 012 e TSKC x (LCR x TR) - 040 também são alternativa ao limoeiro 'Cravo', por induzirem a copa de 'Pêra' à maior tolerância à seca, na fase inicial de produção.

3. Os porta-enxertos limoeiros 'Cravo CNPMF-04' e 'Rugoso Vermelho', citrandarim 'Indio', e híbridos TSKFL x CTARG - 028, TSKC x CTQT1434 - 001, TSKC x CTSW - 058 e LVK x LCR - 038 destacam-se por induzir a copa de 'Pêra CNPMF D6' à precocidade produtiva, semelhantemente ao limão 'Cravo' comum. 


\section{Referências}

AULER， P.A.M.; FIORI-TUTIDA，A.C.G.; TAZIMA， Z.H. Comportamento da laranjeira 'Valência' sobre seis porta-enxertos no noroeste do Paraná. Revista Brasileira de Fruticultura, v.30, p.229-234, 2008. DOI: 10.1590/S0100-29452008000100042.

BARBOSA, C.J.; RODRIGUES, A.S. Tristeza dos citros. Revista Brasileira de Fruticultura, v.36, p.i, 2014. DOI: 10.1590/0100-2945-36-31/13.

BATES, L.S.; WALDREN, R.P.; TEARE, I.D. Rapid determination of free proline for water-stress studies. Plant and Soil, v.39, p.205-207, 1973. DOI: 10.1007/BF00018060.

BLUM, A. Effective use of water (EUW) and not water-use efficiency (WUE) is the target of crop yield improvement under drought stress. Field Crops Research, v.112, p.119-123, 2009. DOI: 10.1016/j.fcr.2009.03.009.

BLUMER, S. Citrandarins e outros híbridos de trifoliata como porta-enxertos nanicantes para a laranjeira 'Valência' (Citrus sinensis L. Osbeck). 2005. 118p. Tese (Doutorado) - Universidade de São Paulo, Piracicaba.

CAMPOS, M.K.F. de; CARVALHO, K. de; SOUZA, F.S. de; MARUR, C.J.; PEREIRA, L.F.P.; BESPALHOK FILHO, J.C.; VIEIRA, L.G.E. Drought tolerance and antioxidant enzymatic activity in transgenic 'Swingle' citrumelo plants over-accumulating proline. Environmental and Experimental Botany, v.72, p.242-250, 2011. DOI: 10.1016/j.envexpbot.2011.03.009.

CARVALHO, K. de; CAMPOS, M.K.F. de; DOMINGUES, D.S.; PEREIRA, L.F.P.; VIEIRA, L.G.E. The accumulation of endogenous proline induces changes in gene expression of several antioxidant enzymes in leaves of transgenic Swingle citrumelo. Molecular Biology Report, v.40, p.3269-3279, 2013. DOI: 10.1007/s11033-012-2402-5.

CHRISTMANN, A.; WEILER, E.W.; STEUDLE, E.; GRILL, E. A hydraulic signal in root-to-shoot signalling of water shortage. The Plant Journal, v.52, p.167-174, 2007. DOI: 10.1111/j.1365-3 13X.2007.03234.x.

CRISTOFANI-YALY, M.; BASTIANEL, M.; FALDONI, L.; BLUMER, S.; POMPEU JUNIOR, J.; CAMPOS, T.M. de P.; SANTOS JÚNIOR, J.A; MACHADO, M.A. dos. Seleção de citrandarins (tangerina Sunki vs. Poncirus trifoliata) para porta-enxertos de citros. Laranja, v.28, p.71-79, 2007.

CUNHA SOBRINHO, A.P. da; PASSOS, O.S.; SOARES FILHO, W. dos S. Cultivares porta-enxerto. In: CUNHA SOBRINHO, A.P. da; MAGALHÃES, A.F. de J.; SOUZA, A. da S.; PASSOS, O.S.; SOARES FILHO, W. dos S. (Ed.). Cultura dos citros. Brasília: Embrapa, 2013. v.1, p.233-292.

DONATO, S.L.R.; PEREIRA, C.S.; BARROS, Z. de J.; SIQUEIRA, D.L. de; SALOMÃO, L.C.C. Respostas de combinações de variedades copa e porta-enxerto de citros à deficiência hídrica. Pesquisa Agropecuária Brasileira, v.42, p.1507-1510, 2007. DOI: $10.1590 / \mathrm{S} 0100-204 X 2007001000020$.

FLEXAS, J.; BOTA, J.; GALMÉS, J.; MEDRANO, H.; RIBASCARBÓ, M. Keeping a positive carbon balance under adverse conditions: responses of photosynthesis and respiration to water stress. Physiologia Plantarum, v.127, p.343-352, 2006. DOI: 10.1111/j.1399-3054.2006.00621.x.

FOOD AND AGRICULTURE ORGANIZATION OF THE UNITED NATIONS. FAOSTAT. [Rome]: FAO, 2012. Available at: $<$ http://faostat3.fao.org $>$. Accessed on: 15 Dec. 2014.

INSTITUTO BRASILEIRO DE GEOGRAFIA E ESTATÍSTICA. Banco de Dados Agregados. Sistema IBGE de Recuperação Automática - SIDRA. 2014. Disponível em: <http://www.sidra. ibge.gov.br>. Acesso em: 15 dez. 2014.

MAGALHÃES FILHO, J.R.; AMARAL, L.R. do; MACHADO, D.F.S.P.; MEDINA, C.L.; MACHADO, E.C. Deficiência hídrica, trocas gasosas e crescimento de raízes em laranjeira Valência sobre dois tipos de porta-enxertos. Bragantia, v.67, p.75-82, 2008. DOI: 10.1590/S0006-87052008000100009.

MATTOS JUNIOR, D. de; NEGRI, J.D. de; FIGUEIREDO, J.O. de; POMPEU JUNIOR, J. Citros: principais informações e recomendações de cultivo. 2005. Disponível em $<$ http://www.iac. sp.gov.br/imagem_informacoestecnologicas/43.pdf $>$. Acesso em: 30 jan. 2013.

MEDINA, C.L.; CASTRO, P.R.C.; KLUGE, R.A.; SESTARI, I. Citros. In: CASTRO, P.R.C.; KLUGE, R.A.; SESTARI, I. (Ed.). Manual de fisiologia vegetal: fisiologia de cultivos. Piracicaba: Ceres, 2008. 864p.

MEDINA, C.L.; MACHADO, E.C. Trocas gasosas e relações hídricas em laranjeira 'Valência' enxertada sobre limoeiro 'Cravo' e trifoliata e submetida à deficiência hídrica. Bragantia, v.57, 1998. Não paginado. DOI: 10.1590/S0006-87051998000100002.

MOLINARI, H.B.C.; MARUR, C.J.; BESPALHOK FILHO, J.C.; KOBAYASHI, A.K.; PILLEGGI, M.; LEITE JUNIOR, R.P.; PEREIRA, L.F.P.; VIEIRA, L.G.E. Osmotic adjustment in transgenic citrus rootstock Carrizo citrange (Citrus sinensis Osb. $\mathrm{x}$ Poncirus trifoliata L. Raf.) overproducing proline. Plant Science, v.167, p.1375-1381, 2004. DOI: 10.1016/j.plantsci.2004.07.007.

MORAES, L.A.C.; MOREIRA, A.; PEREIRA, J.C.R. Incompatibility of 'Cleopatra' mandarin rootstock for grafting citrus in Central Amazon, State of Amazonas, Brazil. Revista de Ciências Agrárias, v.54, p.299-306, 2011. DOI: 10.4322/ rca.2012.026.

NOLTE, K.D.; HANSON, A.D.; GAGE, D.A. Proline accumulation and methylation to proline betaine in Citrus: implications for genetic engineering of stress resistance. Journal of the American Society for Horticultural Science, v.122, p.8-13, 1997.

PORTELA, J.C.; LIBARDI, P.L.; LIER, Q.J. van. Retenção da água em solo sob diferentes usos no ecossistema tabuleiros costeiros. Revista Brasileira de Engenharia Agrícola e Ambiental, v.5, p.49-54, 2001. DOI: 10.1590/S1415-43662001000100009.

RAMOS, Y.C.; STUCHI, E.S.; GIRARDI, E.A.; LEÃO, H.C. de; GESTEIRA, A. da S.; PASSOS, O.S.; SOARES FILHO, W. dos S. Dwarfing rootstocks for 'Valencia' sweet orange. Acta Horticulturae, v.1065, p.351-354, 2015.

RODRIGUEZ-GAMIR, J.; PRIMO-MILLO, E.; FORNER, J.B.; FORNER-GINER, M.A. Citrus rootstock responses to water stress. Scientia Horticulturae, v.126, p.95-102, 2010. DOI: 10.1016/j. scienta.2010.06.015. 
SCHINOR, E.H.; CRISTOFANI-YALY, M.; BASTIANEL, M.; MACHADO, M.A. Sunki Mandarin vs Poncirus trifoliata hybrids as rootstocks for Pera sweet orange. Journal of Agricultural Science, v.5, p.190-200, 2013. DOI: 10.5539/jas.v5n6p190.

SUASSUNA, J.F.; FERNANDES, P.D.; NASCIMENTO, R. do; OLIVEIRA, A.C.M. de; BRITO, K.S.A. de; MELO, A.S. de. Produção de fitomassa em genótipos de citros submetidos a estresse hídrico na formação do porta-enxerto. Revista Brasileira de Engenharia Agrícola e Ambiental, v.16, p.1305-1313, 2012. DOI: $10.1590 /$ S1415-43662012001200007.
SZABADOS, L.; SAVOURE, A. Proline: a multifunctional amino acid. Trends in Plant Science, v.15, p.89-97, 2010. DOI: 10.1016/j.tplants.2009.11.009.

TEXEIRA, D.D.C.; AYRES, J.; KITAJIMA, E.W.; DANET, L.; JAGOUEIX-EVEILLARD, S.; SAILLARD, C.; BOVÉ, J.M. First report of a Huanglongbing-like disease of citrus in São Paulo State, Brazil and association of a new Liberibacter species, "Candidatus Liberibacter americanus", with the disease. Plant Disease, v.89, p.107, 2005. DOI: 10.1094/PD-89-0107A.

Recebido em 6 de maio de 2015 e aprovado em 18 de janeiro de 2016 